\title{
Control engineering approaches at Obuda University for physiological problems
}

\author{
Levente Kovács \\ Research and Innovation Center of Óbuda University, Physiological Controls Group \\ Obuda University, Budapest, Hungary \\ kovacs.levente@nik.uni-obuda.hu
}

\begin{abstract}
Medical devices and computerized medical applications are exponentially increasing nowadays. This is actively supported by the EU's personalized healthcare m-Health and e-Health research programs. The core of such researches is represented undoubtedly by the basic field of biomedical engineering, the physiological modeling, simulation and control topic. In 2013 the Physiological Controls Group of the Obuda University was created in order to integrate in a structural form the university researches in this sense and linking them with educational programs as well. The presentation gives an overview of the obtained results focusing on diabetes, tumor and hemodialysis, but presenting biostatistical approaches as well. The results are presented from the perspective of the recently rewarded ERC StG grant of the EU.
\end{abstract}

As healthcare costs are continuously increasing in modern societies, engineering applications represent one of the most important solutions to treat this problem. By the advancement of computer science large healthcare data is available giving direct target to big data researches, cloud and parallel computations, but specific, personalized therapy possibilities as well. The European Union's ongoing research program, the $\mathrm{H} 2020$ focuses on this engineering supported medical challenge i.e. personalized medicine defined by the H2020 Advisory Group as "a medical model using characterization of individuals".

The concept basically is a direct linkage to one of the thirteen subdisciplines of biomedical engineering, physiological and pathophysiological modeling, simulation and control. This field is mostly a basic research oriented branch of biomedical engineering, where the aim is to understand and mathematically describe given physiological mechanisms and human body interactions in order to create the possibility of medical device implementations, medical software development, medical decision support systems or automatic drug delivery protocols. Hence, it supports the cyber-medical system research concept.

In general, physiological modelling and control focuses on two basic problems: describe the physiological process in a mathematical model, detect/choose the corresponding signal being able to manipulate the model and identify the non-measurable ones.

By establishing in 2013 the Physiological Controls Group $^{1}$ (PhysCon), Obuda University envisaged to step in the above mentioned direction and together with the BioTech Research Center of the Obuda University (created in 2012) to colligate and integrate the biomedical researches at the university. Moreover, the renewed
Computer Engineering MSc curricula started a new specialization in medical informatics as well, based on the researches and know-how of the mentioned two biomedical research entities.

The research topics of PhysCon focus on four areas: diabetes control, targeted molecular based automatic tumor control solutions, biostatistics or evidence-based medicine related investigations and control solutions for hemodialysis machines.

The challenge of personalized medicine from control engineering point of view is to formulate a general control framework applicable for most of the cases of the given problem, and inside it adapt a fine tuned solution focusing on the individual needs.

PhysCon gained expertise throughout the years in the general control solution formulation by using modern robust control techniques. This idea lies on hard constraints formulation on the neglected model uncertainties and was first investigated under PhysCon in the artificial pancreas concept. The main idea was to create a general control framework for type 1 (insulindependent) diabetes patients. The developed algorithm was tested in-silico on real patient data of the Hungarian Artificial Pancreas Working Group established by PhysCon together with the Hungarian Diabetes Association.

In the meantime, we have improved our research concept using novel control techniques on Linear Parameter Varying (LPV) modeling and Tensor Product (TP) approaches using linear matrix inequalities (LMI) based robust solutions.

Based on the promising results we have started investigating the applicability of the control solution in other pathophysiological problems as well. This led our orientation to tumor control focusing on antiangiogenic targeted molecular therapy (ATMT). We have formulated our idea as a double optimization control problem: minimizing the tumor volume and minimizing the drug infusion (costs of therapy) i.e. taming cancer. The research concept was recently rewarded by the EU with the most prestigious individual research grant the ERC StG.

The plenary talk will focus on the presentation of the PhysCon researches, highlighting the robust control perspectives.

\section{ACKNOWLEDGEMENT}

The work is supported by the European Research Council Starting Grant ERC-StG 679681.

\section{${ }^{1}$ http://physcon.uni-obuda.hu/?q=en}


Levente Kovács • Control Engineering Approaches at Óbuda University for Physiological Problems 